Research Paper

\title{
MiR-30a-5p promotes cholangiocarcinoma cell proliferation through targeting SOCS3
}

\author{
Jia Wei Zhang*, Xing Wang*, Gao Chao Li, Dong Wang, Sheng Han, Yao Dong Zhang, Chen Huan Luo, \\ Hong Wei Wang, Wang Jie Jiang, Chang Xian Li ${ }^{\bowtie}$, Xiang Cheng $\mathrm{Li}^{\bowtie}$ \\ Hepatobiliary Center, The First Affiliated Hospital of Nanjing Medical University, Key Laboratory of Living Donor Liver Transplantation, Nanjing, Jiangsu \\ Province, China \\ *Equal contributors
}

$\square$ Corresponding authors: Dr. Chang Xian Li, Hepatobiliary Center, First Affiliated Hospital of Nanjing Medical University, 300 Guangzhou Road, Nanjing, Jiangsu Province, China. E-mail: doclicx20@163.com or Prof. Xiang Cheng Li, Hepatobiliary Center, First Affiliated Hospital of Nanjing Medical University, 300 Guangzhou Road, Nanjing, Jiangsu Province, China. E-mail: drxcli@njmu.edu.cn

( ) The author(s). This is an open access article distributed under the terms of the Creative Commons Attribution License (https://creativecommons.org/licenses/by/4.0/). See http:/ /ivyspring.com/terms for full terms and conditions.

Received: 2019.10.23; Accepted: 2020.03.03; Published: 2020.03.26

\begin{abstract}
Background: MicroRNAs (miRNAs) play important roles in the occurrence and development of cancers. In this project, we aimed to explore the role and molecular mechanism of mir-30a-5p in cholangiocarcinoma (CCA).

Materials and Methods: The expression profile and clinical significance of miR-30a-5p in CCA patients were investigated in 31 ICC and 52 ECC patients respectively. The role and mechanism of miR-30a-5p in CCA cells were investigated by up-regulating and inhibiting miR-30a-5p expression in vitro functional study.

Results: The expression of miR-30a-5p was increased in both CCA tissues and cells. The inhibition of miR-30a-5p decreased cell proliferation and induced cell apoptosis while overexpression of miR-30a-5p achieved the opposite effect. Furthermore, SOCS3 was down-regulated in ICC and ECC tissues and negatively regulated by miR-30a-5p. Dual-luciferase reporter assay revealed that co-transfection of miR-30a-5p significantly inhibited the activity of firefly luciferase reporter carrying the wild-type 3'UTR of SOCS3. The inhibition of SOCS3 could largely rescue the inhibitory effect of miR-30a-5p inhibition on CCA cells proliferation. In clinical, up-regulated miR-30a-5p expression was correlated with large tumor size in both ICC and ECC cohorts.

Conclusions: miR-30a-5p promoted CCA cells proliferation through targeting SOCS3. These findings suggested that miR-30a-5p could be a potential therapeutic target.
\end{abstract}

Key words: miR-30a-5p, cholangiocarcinoma, SOCS3, proliferation, apoptosis

\section{Introduction}

Cholangiocarcinoma (CCA) is a highly malignant tumor originating from the bile duct epithelium, which can be categorized as intrahepatic cholangiocarcinoma (ICC), extra cholangiocarcinoma (ECC) according to the anatomical location. CCA is the second most frequent primary hepatobiliary malignancy and its incidence and mortality gradually increase in the last three decades [1, 2]. Surgical resection is the only possible curative treatment for CCA patients, however, the rates of resectability and the long-term outcome after these therapies are less than satisfactory because of the high post-surgical recurrence [3]. Therefore, elucidating the molecular mechanisms of CCA development and progression are urgently necessary to develop more efficient therapies for CCA.

MicroRNAs (miRNAs) are small non-coding RNAs of 20-22 nucleotides, which can silence or inhibit genes by binding to the target sites of messenger RNAs [4]. It is reported that miRNAs are involved in multiple cellular processes, including cell growth, apoptosis, movement, and differentiation. 
More evidence showed that the dysfunction or abnormal expression of miRNAs maybe lead to incidence of human malignancies, and play important roles in tumor growth and metastasis[5, 6]. Furthermore, miRNAs can as the biomarker of the diagnosis and as potential cancer therapeutics [7, 8]. Recently, increasing researches have revealed that miRNAs were powerful regulators in the tumorigenesis of CCA $[9,10]$. The levels of miR-433 and miR-22 were down-regulated in CCA, and restoration of these miRNAs contributed to ciliary restoration and decreased the malignant phenotype of cancer cells by targeting histone deacetylase 6 [9]. Furthermore, miR-21 was highly expressed in CCA and appeared to mediate resistance of CCA cells to HSP90 inhibitors by reducing levels of DNAJB5[10].

The miR-30 family, which contains five members of distinct pre-mature miRNAs (miR-30a, -30b, -30c, $-30 \mathrm{~d},-30 \mathrm{e})$, has been proved to play diverse roles in regulating essential aspects of tumorigenesis, metastasis, chemo-resistance and clinical prognosis in several types of human cancers [11, 12]. A large number of reports have demonstrated that miR-30a-5p, as a member of the mir-30 family, was down-regulated and associated with tumorigenesis and progression of prostate cancer, hepatocellular carcinoma, breast tumor and some other cancers [13-15]. However, it has been reported that overexpression of miR-30a-5p contributed to initiation and progression of some tumors $[12,16]$. However, the expression and role of miR-30a-5p in CCA progression remain unclear.

In the current study, we aimed to explore the role and molecular mechanism of mir-30a-5p in CCA. Firstly, the expression profile of mir-30a-5p was detected in 83 CCA patients, and analyzed the association with clinical characteristics and prognosis of patients. Subsequently, we investigated the functional role and molecular mechanism of mir-30a-5p in CCA through up-regulating or inhibiting the expression of mir-30a-5p in vitro experiments. Together, our study found that mir-30a-5p contributed to the proliferation of CCA cells by directly binding to the downstream target gene SOCS3.

\section{Materials and methods}

\section{Clinical specimens}

From 2015 to 2018, 83 patients underwent surgical resection and pathologically proven CCA (31 ICC and 52 ECC patients respectively) in the First Affiliated Hospital of Nanjing Medical University, China, were included in current study. Inclusion criteria were: Patients with primary diagnosis of CCA between 2015 and 2018, which underwent surgical resection and pathologically proven CCA. Exclusion criteria were: Patients who received neoadjuvant treatment before primary surgery were excluded. The patients combine with hepatocellular carcinoma or other tumors. All specimens were immediately placed in $4 \%$ formaldehyde and RNA later protective solution for further study. All samples were collected after obtaining written informed consent from all patients. The study has been approved by the ethics committee of The First Affiliated Hospital of Nanjing Medical University.

\section{Cell lines culture and transfection}

Four human CCA cell lines (HCCC9810, QBC939, RBE, HUCCT1) and normal human bile duct cell line (HiBEC) were obtained from the Cell Bank of the Chinese Academy of Science (Shanghai, China). The cell lines were cultured in Dulbecco's Modified Eagle's Medium (DMEM) containing 10\% fetal bovine serum (FBS) and 1\% penicillin-streptomycin (all from Winsent Inc., St.Bruno, Quebec, Canada) in a $5 \% \mathrm{CO}_{2}$ humidified incubator at $37^{\circ} \mathrm{C}$. In order to investigate the role of miR-30a-5p in CCA, inhibition and over-expression of miR-30a-5p were induced by miR-30a-5p agomir and miR-30a-5p antagomir respectively. The transfection was conducted using lipofectamine 2000 (Invitrogen) according to the manufacturer's protocol.

\section{Cell viability and colony formation assays}

Cells were seeded at 1000 cells/well in 96-well plates. Cell viability was measured via Cell Counting Kit-8 (CCK-8, Dojindo, Tokyo, Japan) assay at 0, 24, 48,72 and 96 hours in terms of the manufacturer's instructions. Absorbance was detected at $450 \mathrm{~nm}$ by the spectrophotometer (Thermo Scientific, Pittsburgh, PA, USA). $1 \times 10^{4}$ cells were seeded in 6-well plates and cell proliferation was measured by the colonyformation assay. Each well was fixed with methanol for $15 \mathrm{~min}$ and stained with $0.5 \%$ crystal violet for 30 min.

\section{Quantitative real-time PCR}

Total RNA was extracted from CCA tissues and cells with the use of TRIzol reagent (Invitrogen Corp.) The primer sequences involved were as follows: MiR-30a-5p primer-F, 5'-CGCGATGTTGAAACATCC TCGAC-3'; MiR-30a-5p primer-R, 5'-ATCCAGTGCA GGGTCCGAGG-3'; MiR-30a-5p RT primer, 5'-GTCGT ATCCAGTGCAGGGTCCGAGGTATTCGCACTGGA TACGACCTTCCA-3'. SOCS3 primer-F, 5'-TCGCCAC CTACTGAACCCT; SOCS3-3' primer-R, 5'-GGTCCA GGAACTCCCGAAT-3'; The details of RT-PCR were performed according to the manufacturer's instructions. 


\section{Western blot}

A protein extraction reagent kit (Beyotime, Shanghai, China) was utilized to extract total protein from human CCA tissues and cells according to the manufacturer's instructions. Western blotting was done with a modified version of a previous method [17]. Anti-SOCS3 antibody was purchased from ABCAM. The NIH Image J software (National Institutes of Health, Bethesda, MD) was used to make the results visualized. GAPDH was used as an internal loading control.

\section{Apoptosis assay}

The apoptosis assay was performed by using a FITC annexinV apoptosis detection kit with PI (BioLegend, San Diego, CA, USA). The apoptosis assay was examined by a flow cytometry (FACS Calibur; Becton Dickinson, Franklin Lakes,NJ, USA).

\section{Immunohistochemical staining}

The expression of SOCS3 in CCA tissues were detected by immunohistochemical staining (IHC). The details of IHC staining have been described in our previous article [17]. The images were acquired and quantified by light microscopy and NIS-Elements v4.0 software (Nikon, Tokyo, Japan).

\section{Dual- Luciferase reporter assay}

Cells were seeded in 96-well plates and cultured overnight. Luciferase plasmids encoding wild-type or mutant h-SOCS3-3UTR were co-transfected with miR-30a-5p mimics or negative control in terms of the manufacturer's instruction. The luciferase activities were evaluated at $48 \mathrm{~h}$ after transfection using the Luciferase Reporter System (Promega, Madison, WI, USA) in terms of the manufacturer's instruction.

\section{Subcutaneous xenograft}

All animal experiments were approved by approved by the ethics committee of The First Affiliated Hospital of Nanjing Medical University. A total of 10 athymic nude mice (4-6 weeks old) were purchased from Model Animal Research Center of Nanjing University, Nanjing, China. CCA cells $\left(2 \times 10^{6}\right)$ transfected with lentivirus were injected subcutaneously into the flanks of mice to generate xenograft tumors. During the following 4 weeks, tumor size was evaluated every week to plot the tumor growth curve after injections. Tumor volume was calculated with the following formula: Volume $=\left(\mathrm{W}^{2} \times \mathrm{L}\right) / 2$, where $\mathrm{W}$ means tumor width and $\mathrm{L}$ means tumor length.

\section{Statistical analyses}

The significance of differences between groups was evaluated with Student's t-test and $\chi^{2}$ test as appropriate. Two-sided p-values were calculated, and a probability level of less than 0.05 was considered to be statistically significant. All statistical data were carried out using SPSS computer software version 16 (SPSS Inc, Chicago, IL, USA).

\section{Results}

\section{miR-30a-5p was up-regulated in CCA tissues and cells}

To explore the expression profiles of miR-30a-5p in CCA patients, we first examined the expression in CCA patients from public TCGA database. The data showed that compared to para-tumor, miR-30a-5p was up-regulated in CCA tumor tissues (Fig. 1A-B). To confirm the data from public database, miR-30a-5p levels were measured by quantitative RT-PCR in 31 paired ICC and 52 paired ECC samples. Our results showed that the expression of miR-30a-5p was over-expressed in ICC and ECC tumor tissues respectively compared with their corresponding para-tumor tissues (Fig.1C-D). Furthermore, we also detected the expression profiles of miR-30a-5p in CCA cells. The data showed that the expression of miR-30a-5p was elevated in CCA cell lines compared to the normal bile duct cells line HiBEC (Fig. 1E). Collectively, these results suggested that miR-30a-5p was high expression in both human CCA tissues and cells.

\section{miR-30a-5p promoted CCA cells proliferation}

To determine the role of miR-30a-5p in CCA cells, we used miR-30a-5p antagomir and agomir to inhibit miR-30a-5p in REB cell line (with relatively high miR-30a-5p level) and up-regulate miR-30a-5p in HCCC9810 cell line (with relatively low miR-30a-5p level) respectively (Fig. 2A-B). The results showed that the inhibition of miR-30a-5p significantly inhibited the proliferation of CCA cells while the over-expression of miR-30a-5p promoted the cell proliferation (Fig. 2C-D). Moreover, we also detected the role of miR-30a-5p in cell colony formation. Our data showed that compared to control group, the up-regulation of miR-30a-5p promoted the colony formation. In contrast, the colony number and size were significantly lower in miR-30a-5p inhibited group (Fig. 2E-F). These data indicated that the miR-30a-5p contributed to CCA cells proliferation.

\section{miR-30a-5p inhibited the apoptosis and promoted tumorigenicity of CAA cells}

In order to further investigate the functional role of miR-30a-5p in CCA, cell apoptosis was detected in vitro functional study. The date showed that the inhibition of miR-30a-5p contributed to the apoptosis of CCA cells while up-regulation of miR-30a-5p 
inhibited CCA cells apoptosis (Fig. 3A-B). To further determine the effect of miR-30a-5p on tumorigenicity, xenograft tumor model in nude mice was applied. Our results showed that compared to the control group, the inhibition of miR-30a-5p was able to significantly suppress tumorigenicity resulting in obvious reductions in tumor weight and volume (Fig. 3C-F). These data suggested that miR-30a-5p inhibited CCA cells apoptosis and promoted CCA tumorigenicity.

\section{SOCS3 was down-regulated in CCA tissues and cell lines}

To further explore the mechanism of miR-30a-5p in promoting CCA cells proliferation and tumorigenicity, we used the biological predict software to predict the potential target genes of miR-30a-5p (Fig. 4A). The predict results showed that there were about 63 candidate genes of miR-30a-5p. Among of them, SOCS3 is an important negative feedback regulator in the JAK/STAT pathway. The expression of SOCS3 was further confirmed in CCA samples and cell lines. The results showed that the expression of SOCS3 was down-regulated in CCA cells compared to the normal bile duct cell line HiBEC (Fig. 4B-C). In addition, we further validated the results in the clinical tumor tissues from ICC and ECC patients. Our results showed that the expression of SOCS3 was decreased in ICC and ECC tumor tissues respectively compared with their corresponding para-tumor tissues (Fig. 4D-E). IHC staining results also confirmed that the expression of SOCS3 was down-regulated in ICC and ECC tissue samples (Fig. $4 \mathrm{~F}-\mathrm{G})$. These data suggested that the SOCS3 maybe a suppressor gene of CCA.

\section{miR-30a-5p negatively regulated the expression of SOCS3}

We next investigated the expression relationship between miR-30a-5p and SOCS3 in CCA samples. Our data showed that the expression of SOCS3 was negatively correlated with miR-30a-5p expression in ICC and ECC tissue samples (Fig. 5A-B). These results were further confirmed in CCA cell lines. The results
A

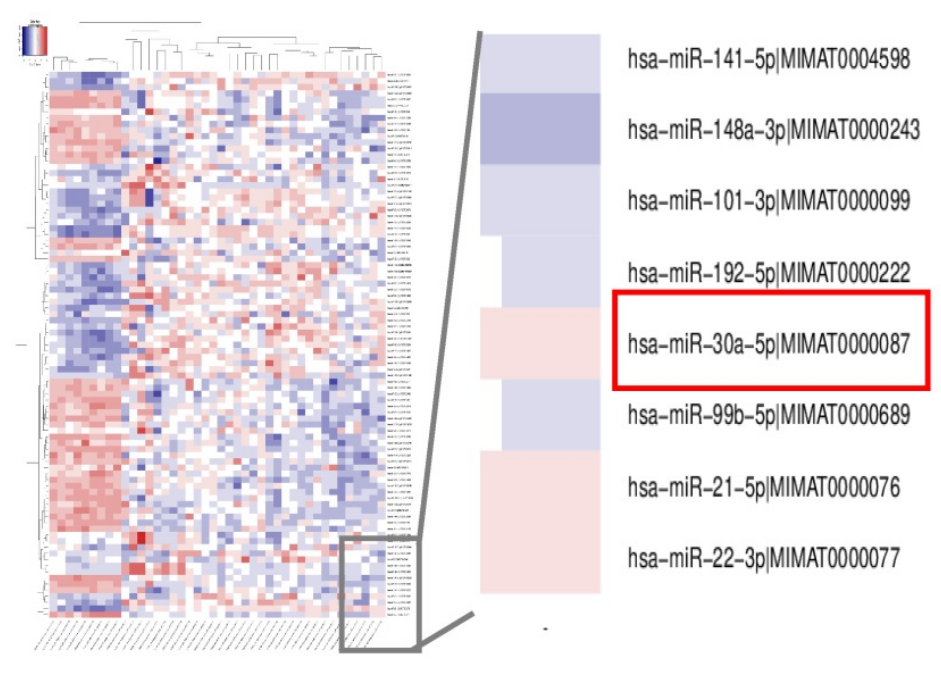

C

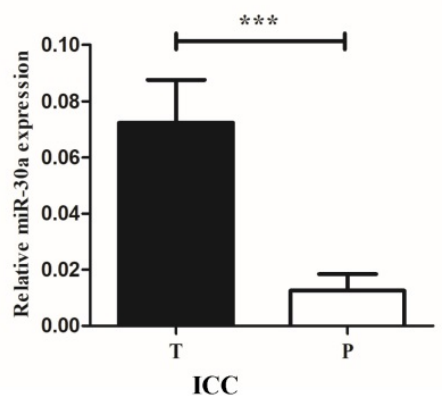

$\mathrm{D}$

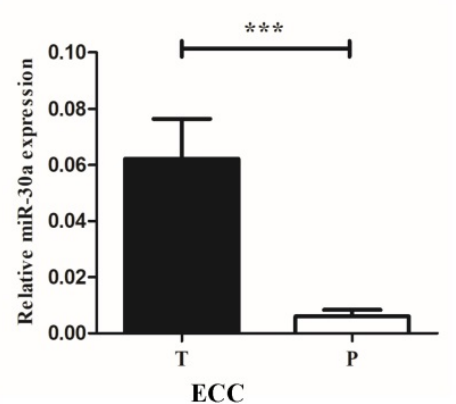

B

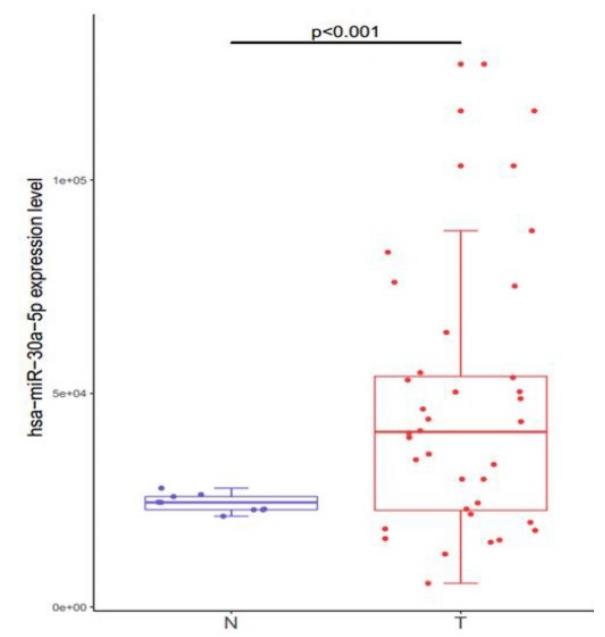

E

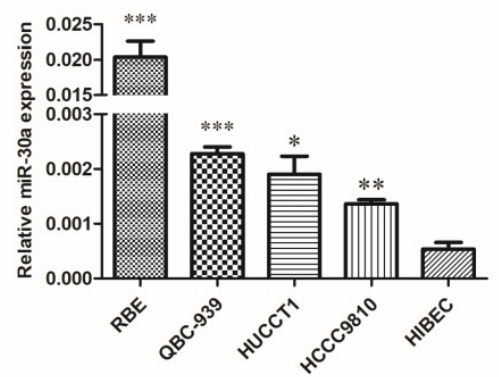

Figure 1. miR-30a-5p was up-regulated in CCA tissues and cells. (A-B) miR-30a-5p was up-regulated in CCA tumor tissues compared to para-tumor in public TCGA database. (C-D) The expression of miR-30a-5p was over-expressed in 31 paired ICC and 52 paired ECC tumor tissues respectively compared with their corresponding para-tumor tissues. (E) The expression of miR-30a-5p was elevated in CCA cells compared to the normal bile duct cells line HiBEC. $* p<0.05$ 
showed that compared to control group, the inhibition of miR-30a-5p increased the expression of SOCS3. In addition, we also found that the expression of SOCS3 was significantly decreased in miR-30a-5p up-regulation group (Fig. 5C-D). After detailed sequence analysis, we observed that 3'UTR of SOCS3 exhibited a putative binding site of miR-30a-5p (Fig.

A

RBE

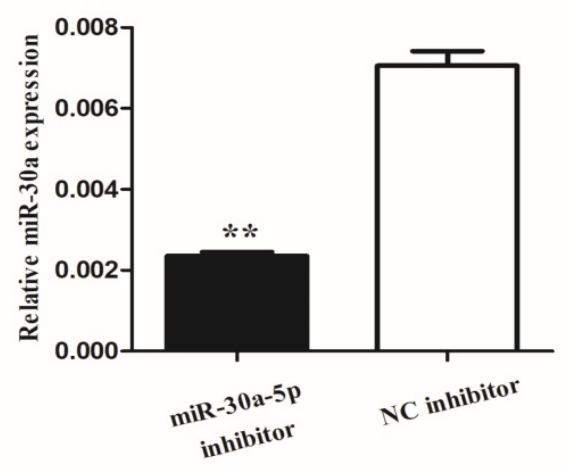

C

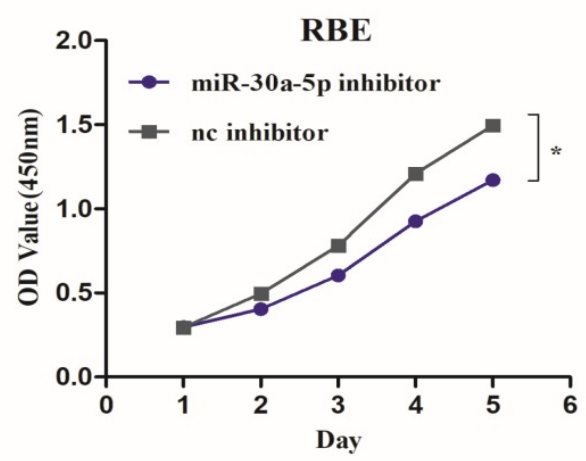

$\mathrm{E}$

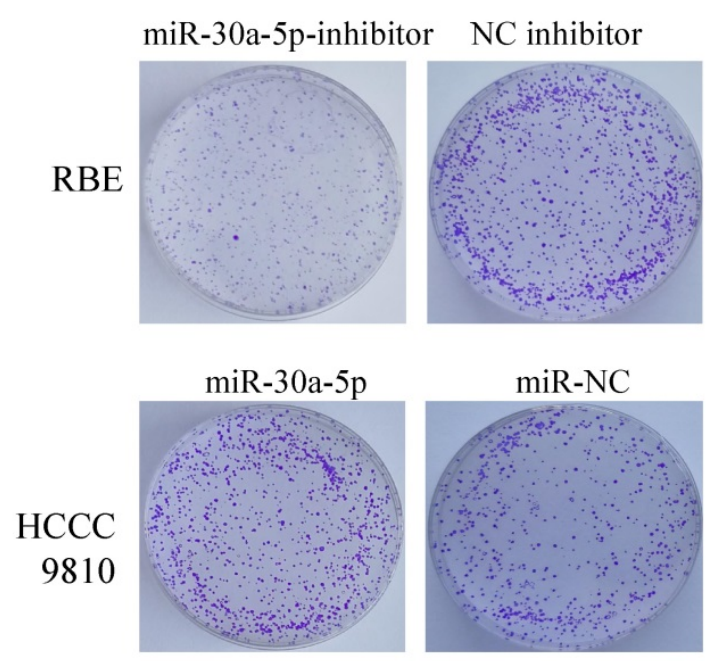

5E). Dual-luciferase reporter assay revealed that co-transfection of miR-30a-5p significantly inhibited the activity of firefly luciferase reporter carrying the wild-type 3'UTR of SOCS3 (Fig. 5F). These data suggested that SOCS3 can be negatively regulated by miR-30a-5p in CCA cells.

B

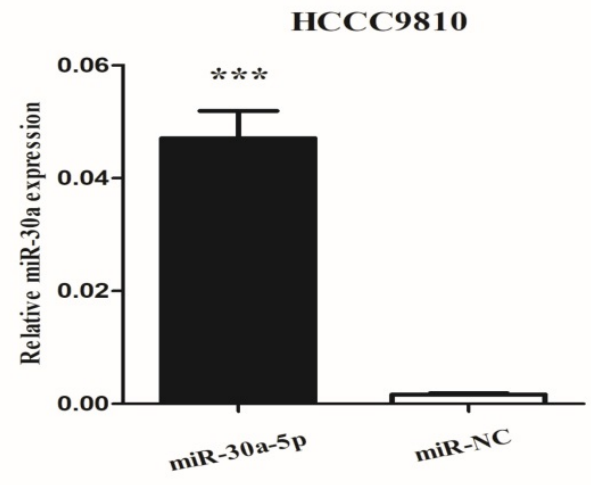

B

$\mathrm{D}$

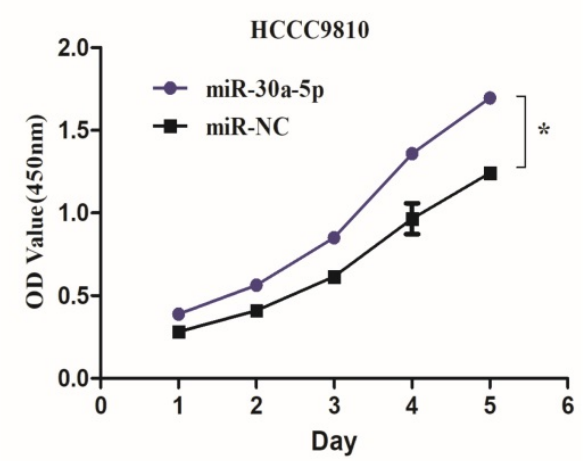

$\mathrm{F}$

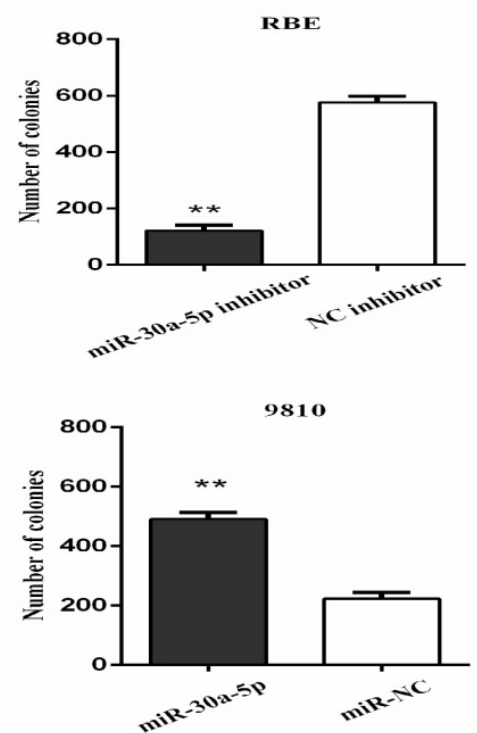

Figure 2. miR-30a-5p promoted CCA cells proliferation. (A-B) miR-30a-5p antagomir and agomir were used to inhibit miR-30a-5p in REB cells and up-regulate miR-30a-5p in HCCC9810 cells respectively. (C-D) The inhibition of miR-30a-5p significantly inhibited the proliferation of CCA cells while the over-expression of miR-30a-5p promoted the cell proliferation. (E-F) The up-regulation of miR-30a-5p promoted the colony formation compared to control group. In contrast, the colony number and size were significantly lower in miR-30a-5p inhibited group. $* p<0.05$ 
A

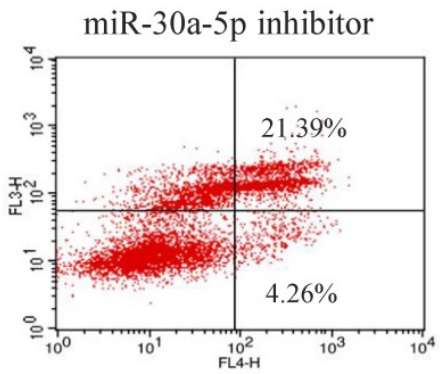

B

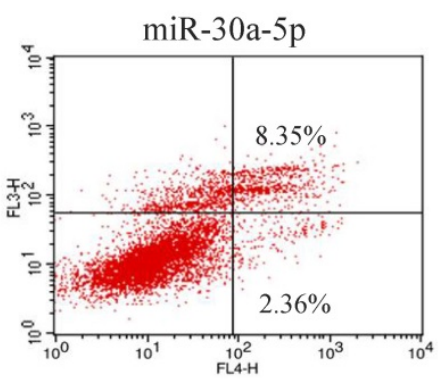

C

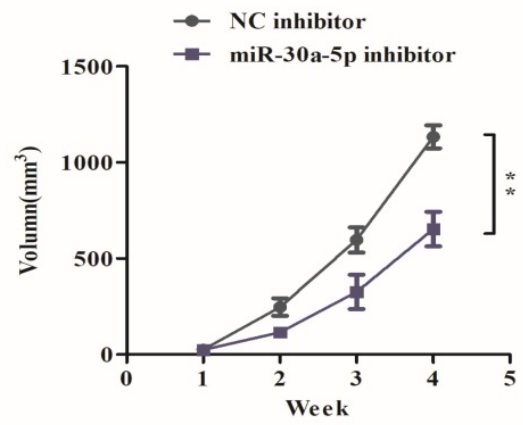

E

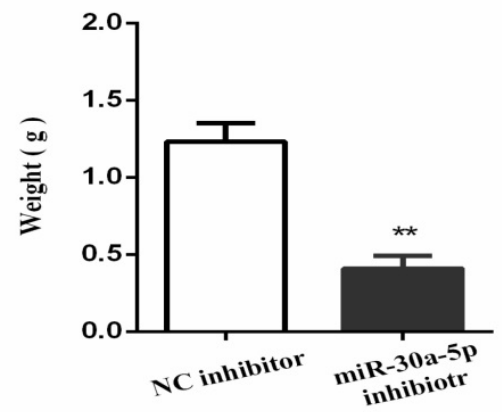

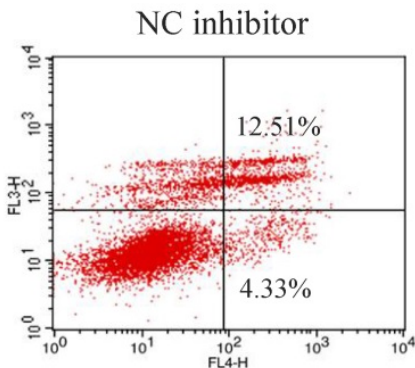
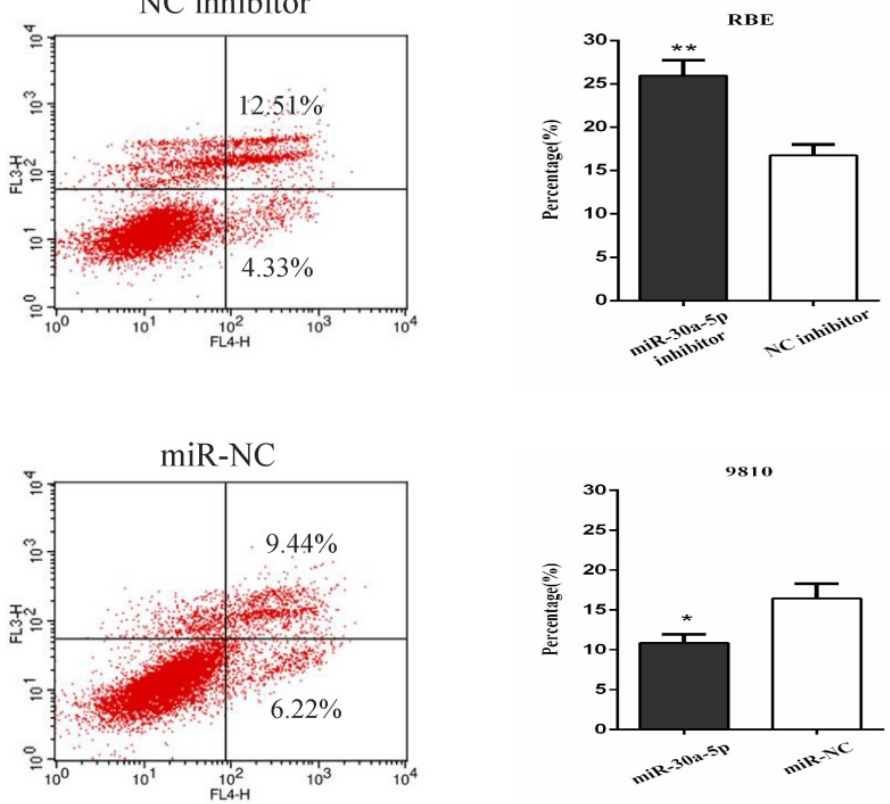

D

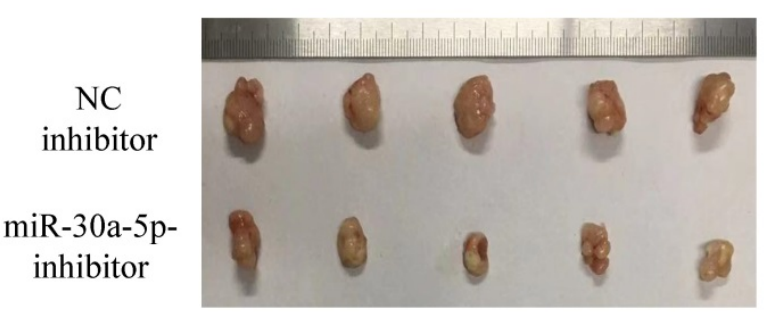

F

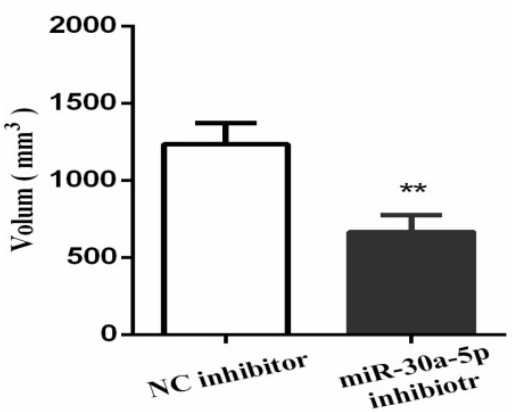

Figure 3. miR-30a-5p inhibited the apoptosis and promoted tumorigenicity of CAA cells. (A-B) The inhibition of miR-30a-5p contributed to the apoptosis of CCA cells while up-regulation of miR-30a-5p inhibited CCA cells apoptosis. (C-F) Compared to the control group, the inhibition of miR-30a-5p was able to significantly suppress tumorigenicity resulting in obvious reductions in tumor weight and volume. ${ }^{*} p<0.05$

\section{miR-30a-5p regulated proliferation of CAA cells in a SOCS3 dependent manner}

To determine whether SOCS3 was key mediator of miR-30a-5p's function in cellular proliferation, SOCS3 was inhibited by shRNA (Fig. 6A). Our results demonstrated that the inhibition of SOCS3 promoted the CCA cells proliferation and colony formation. More importantly, compared to miR-30a-5p inhibition alone, the inhibition of SOCS3 could largely rescue the inhibitory effect of miR-30a-5p inhibition on proliferation and colony formation in CCA cells line (Fig. 6B-C). Furthermore, the inhibition of SOCS3 significantly suppressed cells apoptosis compared to the control group (Fig. 6D). These results indicated that miR-30a-5p regulated proliferation of CAA cell in a SOCS3 dependent manner. 


\section{The clinical significance of miR-30a-5p in CCA patients}

To explore the clinical significance of miR-30a-5p in CCA patients, the correlation of miR-30a-5p expression with clinicopathological parameters of ICC and ECC were analyzed respectively. The results demonstrated that over-expression of miR-30a-5p was positively associated with large tumor size and tumor nodules in ICC cohorts (Table 1). Furthermore, the expression of miR-30a-5p was correlated with tumor size in ECC cohorts (Table 2). Our results also showed that the expression of SOCS3 was negatively correlated with miR-30a-5p expression in ICC and ECC tissue samples. These results collectively suggested that up-regulated miR-30a-5p and lower SOCS3 expressions might have a stimulatory role in the progression of CCA patients.
A

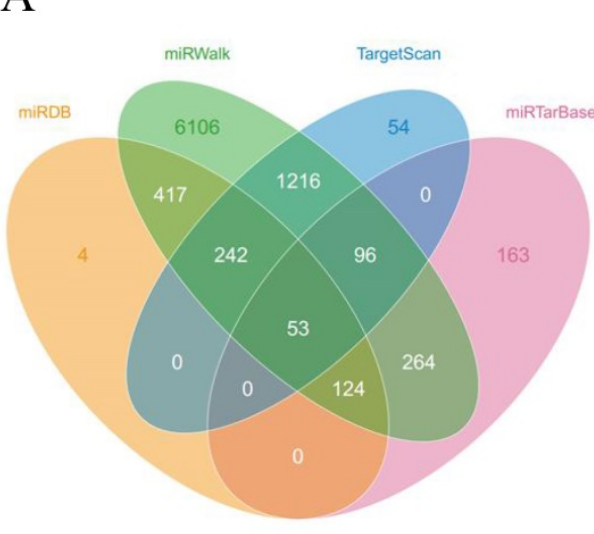

Candidate Genes

\begin{tabular}{|c|c|}
\hline TAF4B & FOXD1 \\
\hline ZNRF1 & EML4 \\
\hline IL1A & SOCS3 \\
\hline MYBL2 & DAG1 \\
\hline MIA3 & SLC7A5 \\
\hline AFF4 & IP6K3 \\
\hline SRSF7 & H6PD \\
\hline
\end{tabular}

B

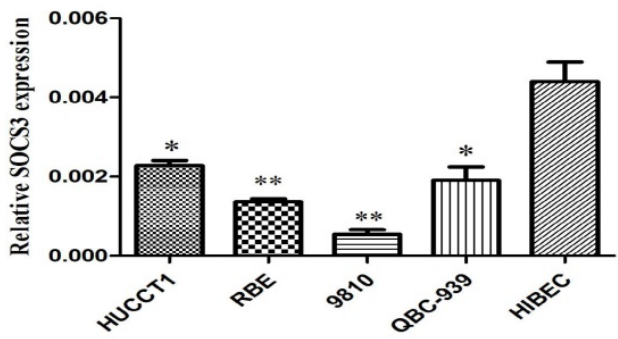

C

SOCS3

GAPDH

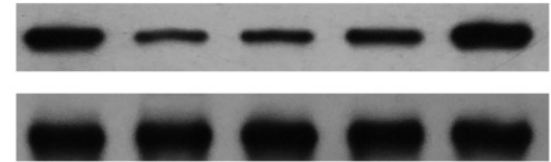

E
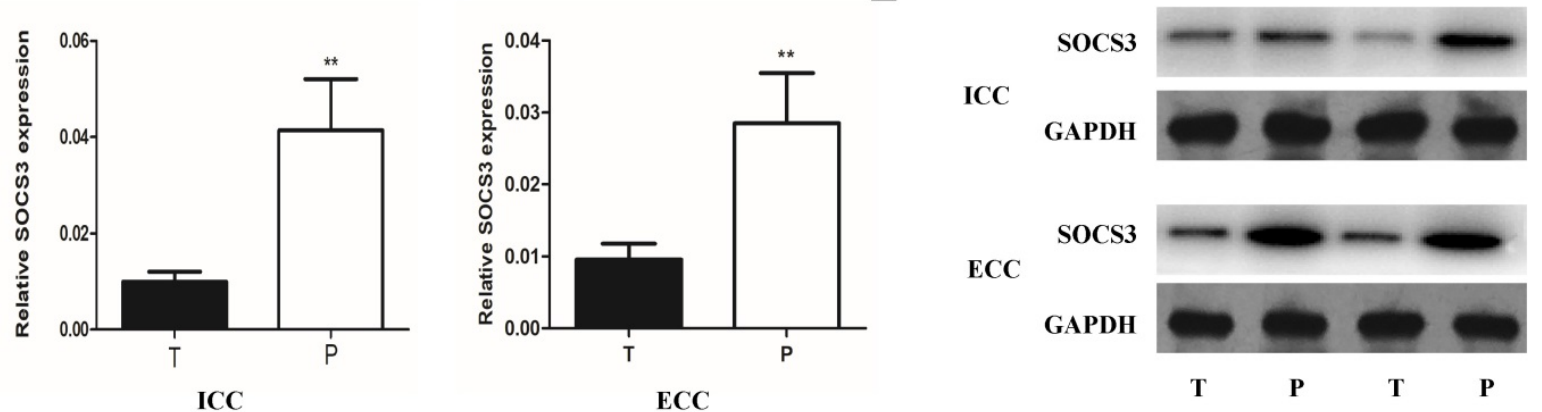

F

G

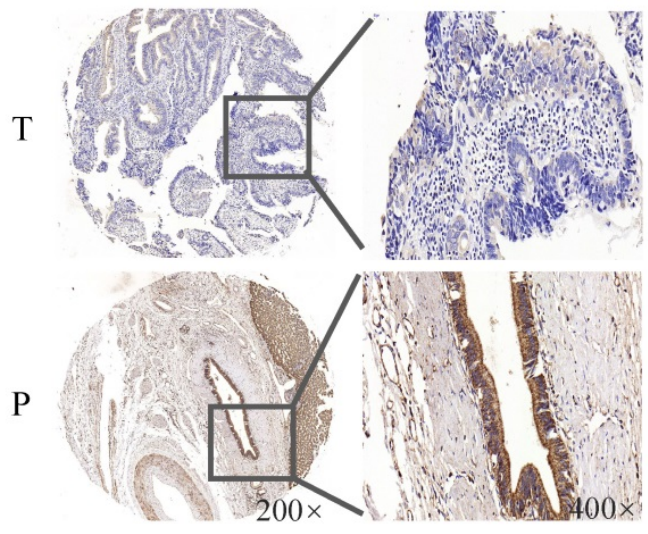

ECC
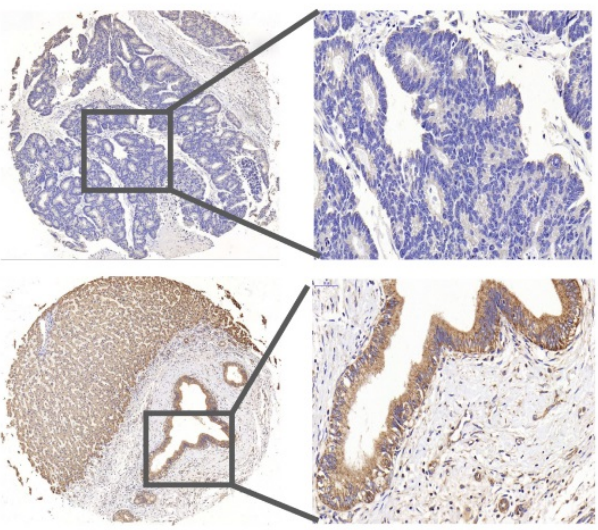

ICC

Figure 4. SOCS3 was down-regulated in CCA tissues and cells. (A) The biological predicted software predicted the potential target genes of miR-30a-5p. (B-C) The expression of SOCS3 was down-regulated in CCA cells compared to the normal bile duct cell line HiBEC. (D-E) The expression of SOCS3 was decreased in ICC and ECC tumor tissues respectively compared with their corresponding para-tumor tissues. (F-G) IHC staining results confirmed that the expression of SOCS3 was down-regulated in ICC and ECC tissue samples. ${ }^{*} p<0.05$ 
A

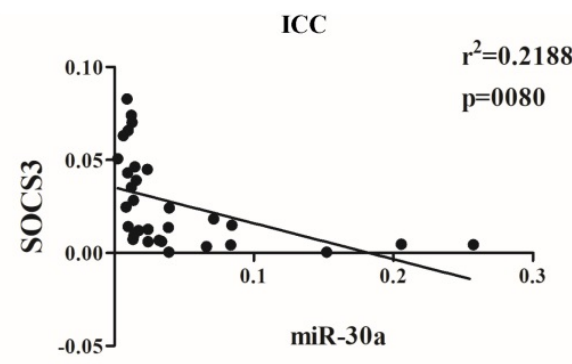

C

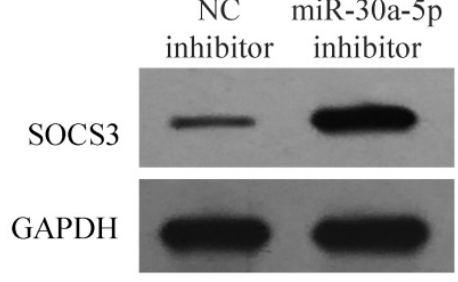

E

SV40 Promoter

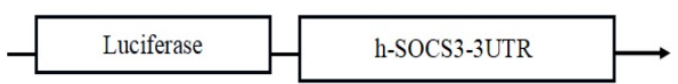

hsa-miR-30a-5p

h-SOCS3-3UTR-wt

h-SOCS3-3UTR-mu
3' ....GAAGGUCAGCUCCUACAAAUGU...5'

| | | | | | |

$5^{\prime} . .$. UUUUUUAAUAAUGUUUACAA .... $3^{\prime}$ $5^{\prime}$....UUUUUUAAUAAGGAUGAGAA.... 3'
B

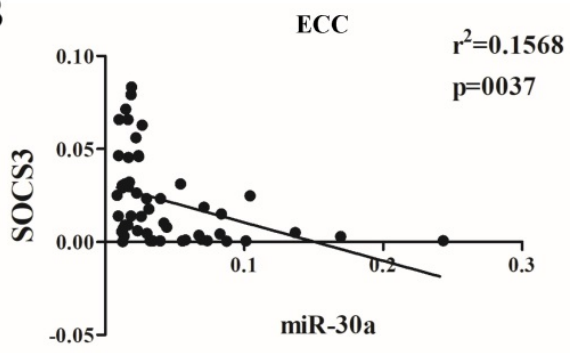

D

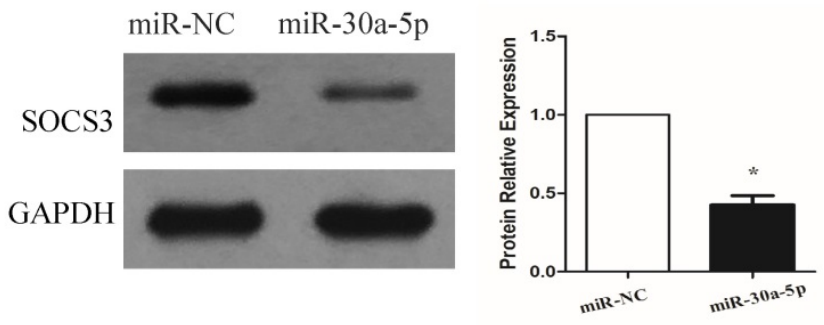

F

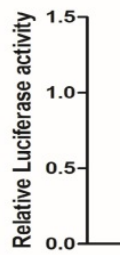

WT-SOCS3 3.UTR MU-SOCS3 3'UTR

miR-30a-NC

miR-30a

Figure 5. miR-30a-5p negatively regulated the expression of SOCS3. (A-B) The expression of SOCS3 was negatively correlated with miR-30a-5p expression in ICC and ECC tissue samples. (C) The inhibition of miR-30a-5p increased the expression of SOCS3. (D) The expression of SOCS3 was significantly decreased in miR-30a-5p up-regulation group. (E) 3'UTR of SOCS3 exhibited a putative binding site of miR-30a-5p. (F) Dual-luciferase reporter assay revealed that co-transfection of miR-30a-5p significantly inhibited the activity of firefly luciferase reporter carrying the wild-type 3'UTR of SOCS3. ${ }^{*} p<0.05$

Table 1. The correlation of miR-30a-5p expression with clinicopathological parameters in ICC patients

\begin{tabular}{|c|c|c|c|c|}
\hline \multirow{2}{*}{$\begin{array}{l}\text { ICC } \\
\text { Clinicopathologic Features }\end{array}$} & \multirow[b]{2}{*}{$\mathbf{n}$} & \multicolumn{3}{|c|}{ miR-30a-5p } \\
\hline & & high & low & $\mathbf{P}$ \\
\hline \multicolumn{5}{|l|}{ Gender } \\
\hline Female & 12 & 6 & 6 & 0.886 \\
\hline Male & 19 & 9 & 10 & \\
\hline \multicolumn{5}{|l|}{ Age } \\
\hline$<60$ & 16 & 7 & 9 & 0.594 \\
\hline$\geq 60$ & 15 & 8 & 7 & \\
\hline \multicolumn{5}{|l|}{ No. of tumor nodules } \\
\hline 1 & 18 & 5 & 13 & $0.022^{*}$ \\
\hline$>1$ & 13 & 9 & 4 & \\
\hline \multicolumn{5}{|l|}{ Tumor size } \\
\hline$<5$ & 11 & 3 & 8 & $0.022^{*}$ \\
\hline$\geq 5$ & 20 & 14 & 6 & \\
\hline \multicolumn{5}{|l|}{ Pathological stages } \\
\hline $\mathrm{I}+\mathrm{II}$ & 9 & 4 & 5 & 0.779 \\
\hline III + IV & 22 & 11 & 11 & \\
\hline \multicolumn{5}{|l|}{ Vascular invasion } \\
\hline Absent & 19 & 11 & 8 & 0.625 \\
\hline Present & 12 & 8 & 4 & \\
\hline \multicolumn{5}{|l|}{ Tumor Stages } \\
\hline $\mathrm{I}+\mathrm{II}$ & 16 & 10 & 6 & 0.632 \\
\hline $\mathrm{III}+\mathrm{IV}$ & 15 & 7 & 8 & \\
\hline
\end{tabular}

Table 2. The correlation of miR-30a-5p expression with clinicopathological parameters in ECC patients

\begin{tabular}{lllll}
\hline ECC & & miR-30a-5p & \\
\cline { 5 - 5 } Clinicopathologic Features & $\mathbf{n}$ & high & low & P \\
\hline Gender & & & & \\
Female & 16 & 6 & 10 & 0.309 \\
Male & 36 & 19 & 17 & \\
Age & & & & \\
$<60$ & 34 & 17 & 17 & 0.703 \\
$\geq 60$ & 18 & 8 & 10 & \\
Tumor size & & & & \\
$<3$ & 32 & 13 & 19 & $0.016^{*}$ \\
$\geq 3$ & 20 & 15 & 5 & \\
Pathological stages & & & & \\
I + II & 23 & 11 & 12 & \\
III + IV & 29 & 11 & 18 & \\
Vascular invasion & & & & \\
Absent & 37 & 11 & 26 & \\
Present & 15 & 8 & 7 & \\
Tumor Stages & & & & \\
I + II & 25 & 10 & 15 & \\
III + IV & 27 & 15 & 12 & \\
\hline
\end{tabular}


A
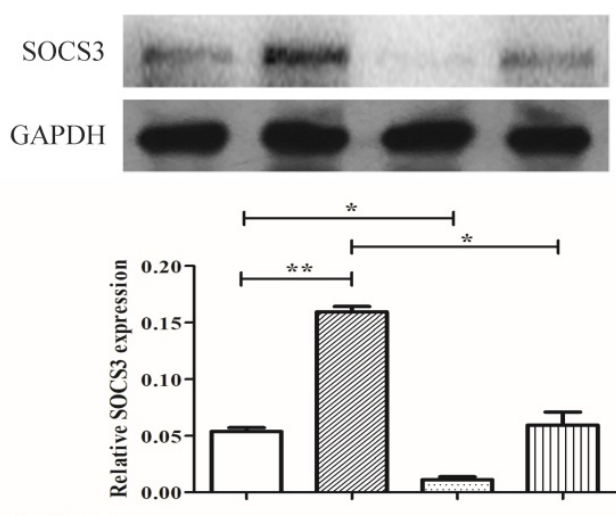

miR-30a-5p inhibitor $\mathrm{NC}$ inhibitor sh-SOCS3 sh-NC

C

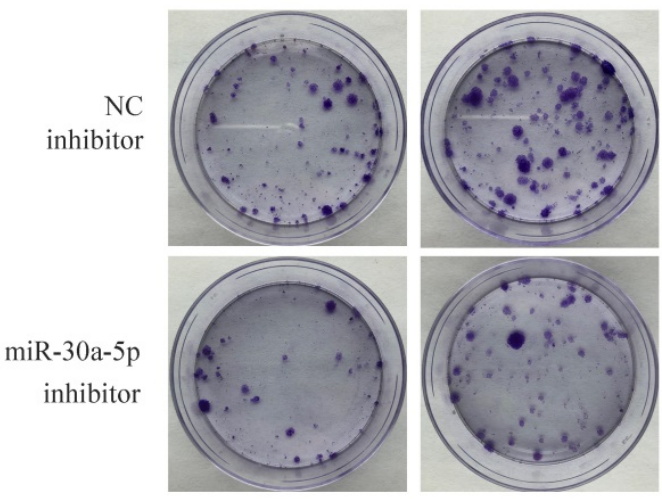

D
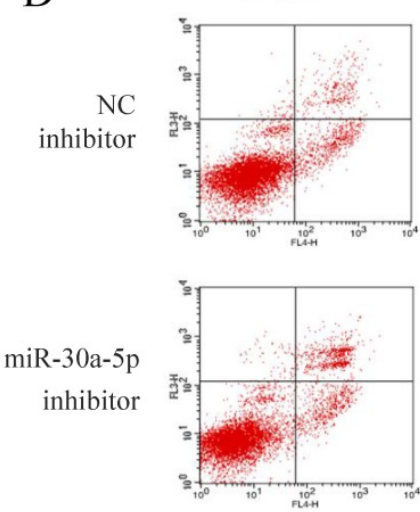

\begin{abstract}
$+$
\end{abstract}
$-$

-
-
+

$-\quad+$

$+$

$+$

B

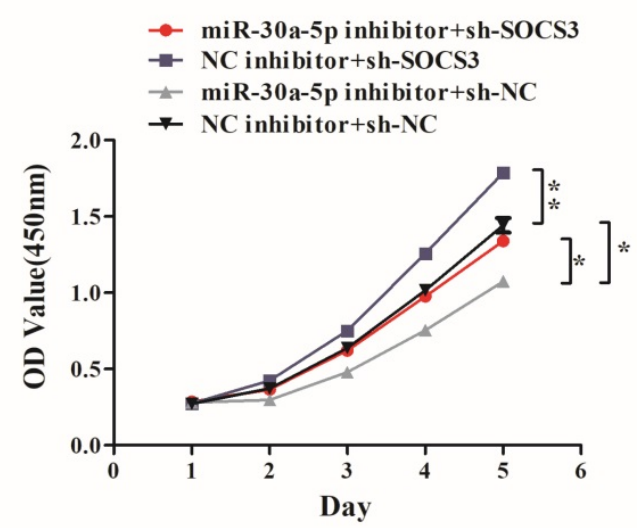

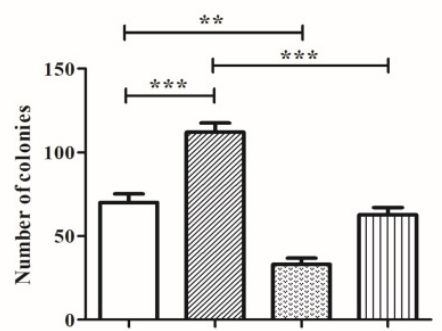

miR-30a-5p inhibitor NC inhibitor sh-NC $++{ }_{-}+$

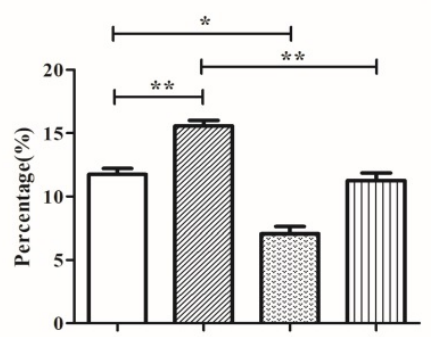

miR-30a-5p inhibitor $\quad-\quad+\quad+\quad-\quad+$

$\mathrm{NC}$ inhibitor $+\quad-\quad+\quad-$

sh-SOCS3 - $\quad-\quad+\quad+$

sh-NC $+\quad+\quad-$

Figure 6. miR-30a-5p regulated proliferation of CAA cells in a SOCS3 dependent manner. (A) The expression of SOCS3 was inhibited by shRNA. (B) Compared to miR-30a-5p inhibition alone, the inhibition of SOCS3 could rescue the inhibitory effect of miR-30a-5p inhibition on proliferation of CCA cells. (C) (B) The inhibition of SOCS3 could rescue the inhibitory effect of miR-30a-5p inhibition on colony formation in CCA cells. (D) The inhibition of SOCS3 significantly suppressed cells apoptosis compared to the control group. $* p<0.05$

\section{Discussion}

To our knowledge, this is the first report on the roles and mechanisms of miR-30a-5p in CCA. In this study, our results demonstrated that the expression of miR-30a-5p was increased in both CCA tissues and cells. It was consistent with previous data displayed in the TCGA database. Furthermore, overexpression of miR-30a-5p was significantly associated with tumor size and nodules of CCA patients. The inhibition of miR-30a-5p suppressed tumor cell proliferation and induced cell apoptosis. Collectively, these data suggested that miR-30a-5p could be a potential therapeutic target of CCA. 
miR-30a-5p, as a member of the mir-30 family, plays a crucial role in tumor growth and metastasis [5, 6]. Currently, the expression profile and function of miR-30a-5p in different malignancies are still existed controversies. A large number of reports have demonstrated that miR-30a-5p was commonly down-expression and regulated biological behaviors of tumor growth, invasion, epithelial-mesenchymal transition (EMT) in various types of cancers [13, 14, 18]. miR-30a-5p was down-regulated in prostate cancer and inhibited cell proliferation via targeting PCLAF [14]. Furthermore, the down-regulation of miR-30a-5p was associated with poor prognosis and promoted chemoresistance of gemcitabine in pancreatic ductal adenocarcinoma [19]. However, it has been also reported that overexpression of miR-30a-5p contributed to initiation and progression of some tumors $[12,16]$. miR-30a-5p was increased and as a potential biomarker for ovarian serous adenocarcinoma[16]. In addition, the expression of miR-30a-5p was increased in glioma tissues and promoted glioma cell proliferation, migration, and invasion via targeting WWP1 [12]. These consistent with our present data. miR-30a-5p was over-expressed in CCA samples and cells. The inhibition of miR-30a-5p suppressed tumor cell proliferation and induced cell apoptosis of CCA.

Suppressor of cytokine signaling (SOCS) proteins, as negative feedback regulators of cytokine signaling, are induced by interleukins and various peptide hormones and may prevent sustained activation of signaling pathways [20]. SOCS3, a member of SOCS family, acts as a negative feedback regulator of the Janus-activated kinase/signal transducers and activators of transcription factor (JAK/STAT) signaling pathway [21, 22]. More evidence showed that abnormal expression of SOCS3 is observed in a variety of malignant tumors, which may lead to promoter methylation and further contribute to the occurrence and development of tumors [23, 24]. Hajime et al showed that reconstitution of SOCS3 expression reduced the duration and magnitude of IL-6-mediated STAT-3 phosphorylation, decreased cellular Mcl-1 levels, and sensitized CCA cells to TRAIL-induced killing [25]. Furthermore, overexpression of SOCS3 inhibited the IL-6-induced epithelial-to-mesenchymal transition and CCA cell metastasis [26]. Recently, increasing research showed that microRNA regulated tumor development and progression through targeting SOCS expression[27, 28]. However, whether miR-30a-5p promoted CCA cells proliferation by regulating SOCS3 has not been reported. In this study, the expression of SOCS3 was up-regulated in miR-30a-5p -inhibition CCA cell lines. Furthermore, the IHC results in CCA patients showed that there was significantly negatively association between miR-30a-5p with SOCS3. More interesting, the inhibition of SOCS3 could largely rescue the inhibitory effect of miR-30a-5p inhibition on proliferation of CCA cells. Dual-luciferase reporter assay revealed that co-transfection of miR-30a-5p significantly inhibited the activity of firefly luciferase reporter carrying the wild-type $3^{\prime} U T R$ of SOCS3. These data demonstrated that miR-30a-5p negatively regulated the SOCS3 signaling to exert its tumor-promoted functions in CCA.

In summary, we demonstrated that miR-30a-5p was up-regulation and significantly associated with tumor size and nodules of CCA patients. The inhibition of miR-30a-5p decreased cell proliferation and induced cell apoptosis while overexpression of miR-30a-5p achieved the opposite effect. Furthermore, we also showed that miR-30a-5p promoted CCA progression through regulating SOCS3 signaling pathway. Together, miR-30a-5p could be a potential therapeutic target for CCA. A larger sample size is needed to further explore the clinical significance of miR-30a-5p in CCA patients.

\section{Abbreviations}

MicroRNAs (miRNAs), Cholangiocarcinoma (CCA), Intrahepatic cholangiocarcinoma (ICC), Extra cholangiocarcinoma (ECC), Suppressor of cytokine signaling (SOCS).

\section{Acknowledgments}

This study was supported by National Science Foundation of China (NSFC) (81700572, 81670570), Natural Science Foundation of Jiangsu Province, China (BK20171077) and Key research and development program of Jiangsu Province (BE2016789).

\section{Author Contributions}

$\mathrm{XC} \mathrm{Li}$ and $\mathrm{CX} \mathrm{Li}$ were involved in the study design. JW Zhang, X Wang, GC Li, D Wang, S Han, YD Zhang, HW Wang, CH Luo and WJ Jiang were involved in investigation, analysis and manuscript preparation. All authors read and approved the final manuscript.

\section{Competing Interests}

The authors have declared that no competing interest exists.

\section{References}

1. Global Burden of Disease Cancer C, Fitzmaurice C, Dicker D et al: The Global Burden of Cancer 2013. JAMA Oncol 2015, 1:505-527.

2. Khan SA, Davidson BR, Goldin RD et al: Guidelines for the diagnosis and treatment of cholangiocarcinoma: an update. Gut 2012, 61:1657-1669.

3. Cillo U, Fondevila C, Donadon M et al: Surgery for cholangiocarcinoma. Liver Int 2019, 39 Suppl 1:143-155. 
4. Bartel DP: MicroRNAs: target recognition and regulatory functions. Cell 2009, 136:215-233.

5. Akpa MM, Iglesias D, Chu L et al: Wilms Tumor Suppressor, WT1, Cooperates with MicroRNA-26a and MicroRNA-101 to Suppress Translation of the Polycomb Protein, EZH2, in Mesenchymal Stem Cells. J Biol Chem 2016, 291:3785-3795.

6. Li Y, Chen D, Su Z et al: MicroRNA-106b functions as an oncogene in renal cell carcinoma by affecting cell proliferation, migration and apoptosis. Mol Med Rep 2016, 13:1420-1426.

7. Liu R, Chen X, Du Y et al: Serum microRNA expression profile as a biomarker in the diagnosis and prognosis of pancreatic cancer. Clin Chem 2012, 58:610-618.

8. Trang P, Weidhaas JB, Slack FJ: MicroRNAs as potential cancer therapeutics. Oncogene 2008, 27 Suppl 2:S52-57.

9. Mansini AP, Lorenzo Pisarello MJ, Thelen KM et al: MicroRNA (miR)-433 and miR-22 dysregulations induce histone-deacetylase- 6 overexpression and ciliary loss in cholangiocarcinoma. Hepatology 2018, 68:561-573.

10. Lampis A, Carotenuto P, Vlachogiannis G et al: MIR21 Drives Resistance to Heat Shock Protein 90 Inhibition in Cholangiocarcinoma. Gastroenterology 2018, 154:1066-1079 e1065.

11. Liu MM, Li Z, Han XD et al: MiR-30e inhibits tumor growth and chemoresistance via targeting IRS1 in Breast Cancer. Sci Rep 2017, 7:15929.

12. Zhao $\mathrm{P}$, Wang $\mathrm{M}$, An J et al: A positive feedback loop of miR-30a-5p-WWP1-NF-kappaB in the regulation of glioma development. Int J Biochem Cell Biol 2019, 112:39-49.

13. Li L, Kang L, Zhao W et al: miR-30a-5p suppresses breast tumor growth and metastasis through inhibition of LDHA-mediated Warburg effect. Cancer Lett 2017, 400:89-98.

14. Zhao H, Lai $X$, Zhang $W$ et al: MiR-30a-5p frequently downregulated in prostate cancer inhibits cell proliferation via targeting PCLAF. Artif Cells Nanomed Biotechnol 2019, 47:278-289.

15. Huang WT, Chen ZX, He RQ et al: Clinicopathological role of miR-30a-5p in hepatocellular carcinoma tissues and prediction of its function with bioinformatics analysis. Onco Targets Ther 2016, 9:5061-5071.

16. Zhou J, Gong G, Tan H et al: Urinary microRNA-30a-5p is a potential biomarker for ovarian serous adenocarcinoma. Oncol Rep 2015, 33:2915-2923.

17. Li CX, Ng KT, Shao Y et al: The inhibition of aldose reductase attenuates hepatic ischemia-reperfusion injury through reducing inflammatory response. Ann Surg 2014, 260:317-328.

18. Zhong Z, Xia Y, Wang P et al: Low expression of microRNA-30c promotes invasion by inducing epithelial mesenchymal transition in non-small cell lung cancer. Mol Med Rep 2014, 10:2575-2579.

19. Zhou L, Jia S, Ding G et al: Down-regulation of miR-30a-5p is Associated with Poor Prognosis and Promotes Chemoresistance of Gemcitabine in Pancreatic Ductal Adenocarcinoma. J Cancer 2019, 10:5031-5040.

20. Yoshimura A, Naka T, Kubo M: SOCS proteins, cytokine signalling and immune regulation. Nat Rev Immunol 2007, 7:454-465.

21. Shuai K, Liu B: Regulation of JAK-STAT signalling in the immune system. Nat Rev Immunol 2003, 3:900-911.

22. Croker BA, Kiu H, Nicholson SE: SOCS regulation of the JAK/STAT signalling pathway. Semin Cell Dev Biol 2008, 19:414-422.

23. Fu X, Ren L, Chen J et al: Characterization of the roles of suppressor of cytokine signaling-3 in prostate cancer development and progression. Asia Pac J Clin Oncol 2015, 11:106-113.

24. Zhang X, You Q, Zhang X et al: SOCS3 Methylation Predicts a Poor Prognosis in HBV Infection-Related Hepatocellular Carcinoma. Int J Mol Sci 2015, 16:22662-22675.

25. Isomoto H, Mott JL, Kobayashi $\mathrm{S}$ et al: Sustained IL-6/STAT-3 signaling in cholangiocarcinoma cells due to SOCS-3 epigenetic silencing. Gastroenterology 2007, 132:384-396.

26. Zhou QX, Jiang XM, Wang ZD et al: Enhanced expression of suppresser of cytokine signaling 3 inhibits the IL-6-induced epithelial-to-mesenchymal transition and cholangiocarcinoma cell metastasis. Med Oncol 2015, 32:105.

27. Xu CH, Liu Y, Xiao LM et al: Silencing microRNA-221/222 cluster suppresses glioblastoma angiogenesis by suppressor of cytokine signaling-3-dependent JAK/STAT pathway. J Cell Physiol 2019, 234:22272-22284.

28. Zhou X, Yan T, Huang C et al: Melanoma cell-secreted exosomal miR-155-5p induce proangiogenic switch of cancer-associated fibroblasts via SOCS1/ JAK2/STAT3 signaling pathway. J Exp Clin Cancer Res 2018, 37:242. 